\title{
Influência do vinhoto no desenvolvimento de Stomoxys calcitrans
}

\author{
Influence of vinasse on the development of Stomoxys calcitrans
}

\section{Isabela Helena Ferreira Leite ${ }^{\mathrm{I}}$ Éder Batista de Carvalho ${ }^{\mathrm{II}}$ Avelino José Bittencourt $^{\mathrm{III}}$}

\section{RESUMO}

\begin{abstract}
Stomoxys calcitrans (Linnaeus, 1758) (Diptera: Muscidae) é conhecida por sua dolorosa picada, que causa estresse nos animais, e pela transmissão de diversas enfermidades, além de atacar o homem. Devido aos surtos ocorridos em áreas sucroalcooleiras no Brasil, onde o vinhoto é utilizado na fertirrigação dos canaviais, elaborou-se o presente estudo, que teve como objetivo verificar se o vinhoto favorece a postura e o desenvolvimento dos estádios imaturos desse inseto. Para tanto, ovos foram depositados em dieta de crescimento larval de Bailey (controle) e também em dietas com substituição de 5, 10, 20 e 30\% do seu volume de água por vinhoto. Parte dos resultados foi submetida à analise de variância e as médias foram comparadas entre si pelo teste de Tukey. A outra parte foi avaliada pelo teste do Qui Quadrado. A viabilidade dos ovos foi de 66\%, 64\%, 69\%, 80\% e $20 \%$ das larvas de 96,4\%, 96,9\%, 95,1\%, 99,3\% e 97,2\% e das pupas de $90,2 \%, 94,7 \%, 93,5 \%, 91,5 \%$ e $86,1 \%$, respectivamente, para os grupos controle, $5,10,20$, e 30\%. O peso das larvas de terceiro estágio foi de 16,4; 16,9; 19,7; 15,8 e 18mg, e das pupas foi 12,9; 13,9;17,4; 14,5 e 21,9mg. O período larval foi de 10,38, 11,81, 10,00, 10,86, 9,5 dias, e o pupal foi de 6,$57 ; 6,19 ; 7,05 ; 7,00$ e 6,00, respectivamente. A viabilidade dos estádios imaturos foi maior nas dietas com substituição de vinhoto. Algumas concentrações de vinhoto utilizadas nas dietas afetaram a biologia dos estádios imaturos de $\boldsymbol{S}$. calcitrans.
\end{abstract}

Palavras-chave: cana de açúcar, vinhoto, mosca dos estábulos.

\section{ABSTRACT}

Stomoxys calcitrans is known for its painful sting that causes stress in animals and also by transmitting several diseases, and attack man. Due to the outbreaks in sugarcane fields in Brazil, where the vinasse is used in fertigation of the sugarcane fields, this study was conducted, aiming to verify if the vinasse would improve posture and development of the immature stages of this insect. For this, eggs were deposited into diet of larval growth of Bailey (control), and also in diets with replacement of 5, 10, 20 and 30\% of this water volume per vinasse. Part of the results was submitted to analysis of variance and means were compared by Tuket test, and the other, was assessed by Chi-square. The eggs viability was of $66 \%, 64 \%, 69 \%, 80 \%$ e $20 \%$, the larvae was of $96.4 \%, 96 ., 9 \%$, $95.1 \%, 99.3 \%$ e $97.2 \%$ and the pupae was of $90.2 \%, 94.7 \%$, $93.5 \%, 91.5 \%$ and $86.1 \%$, respectively to the control, 5, 10, 20 , e 30\%. The weight of the third stage larvae was 16.4; 16.9; 19.7; 15.8 and $18 \mathrm{mg}$, and pupae was of 12.9; 13.9; 17.4; 14.5 e $21.9 \mathrm{mg}$. The larval period was of 10.38, 11.81, 10.00, 10.86, 9.5 days, and the pupal was of 6.57, 6.19, 7.05, 7.00 and 6.00 days, respectively. The viability of the immature stages was higher in diet with replacement of vinasse. Some of vinasse concentrations used in diets affected the biology of immature stages of $\boldsymbol{S}$. calcitrans.

Key words: sugar cane, vinasse, stable fly.

\section{INTRODUÇÃO}

A mosca Stomoxys calcitrans (Linnaeus, 1758) (Diptera: Muscidae), conhecida como mosca dos estábulos, é um díptero hematófago que acomete várias espécies de animais (BISHOPP, 1913) e o homem (KING; LENERT, 1936). Apresenta distribuição geográfica mundial (SOULSBY, 1987) e no Brasil, devido ao seu

\footnotetext{
IDepartamento de Parasitologia Veterinária, Universidade Federal Rural do Rio de Janeiro (UFRRJ), Km 7 da Rodovia Rio - São Paulo, Seropédica, RJ, Brasil. E-mail: isabelahfl@gmail.com. Autor para correspondência.

"Médico Veterinário Autônomo, Santa Rita do Ibitipoca, MG, Brasil.

IIIDepartamento de Medicina e Cirurgia Veterinária, UFRRJ, Seropédica, RJ, Brasil.
} 
clima tropical está presente em várias regiões (MORAES, 1990) principalmente nos meses mais quentes do ano (BITTENCOURT, 1998).

A mosca dos estábulos pode se desenvolver em locais que contenham restos culturais que tenham permanecido no campo por algum tempo, principalmente se esses materiais se encontrarem fermentados ou umedecidos com urina e fezes de gado (GUIMARÃES, 1983). Inclusive os restos alimentares que ficam debaixo dos cochos de alimentação e o vinhoto podem atrair e estimular a postura desse díptero (BURALLI et al., 1987).

$\mathrm{O}$ volume de vinhoto que é produzido e utilizado na fertirrigação, associado à palhada da canade-açúcar que fica no solo após a colheita mecanizada, bem como a capacidade de voo da mosca dos estábulos (BAILEY et al., 1973; HOGSETTE; RUFF, 1985), podem criar condições favoráveis para o aumento da população destes dípteros, pois a matéria orgânica necessária ao desenvolvimento da mosca estaria disponível em grande quantidade, e os animais em que as moscas se alimentam são criados nas proximidades dos canaviais.

O objetivo do presente estudo foi verificar se o vinhoto favorece o desenvolvimento dos estádios imaturos de $\boldsymbol{S}$. calcitrans em condições controladas de temperatura e umidade.

\section{MATERIAL E MÉTODOS}

Moscas adultas foram capturadas com redes entomológicas, levadas ao laboratório para identificação, segundo FURMAN e CATS (1982), e transferidas para gaiolas de criação. Todos os estádios foram mantidos em estufa tipo B. O. D. para entomologia à temperatura média de $27^{\circ} \mathrm{C}$, umidade relativa de $80 \%$ e foto período de 24 horas. Diariamente, foram alimentadas com sangue bovino citratado $0,38 \%$ (BENIGNO, 1987), aquecido em banho-maria e fornecido em placa de Petri com almofada de gaze, para obtenção dos ovos.

Para o desenvolvimento das fases imaturas, utilizou-se a dieta descrita por BAILEY (1973) e, para a realização deste experimento, foi utilizada esta dieta com substituição de água por vinhoto nas concentrações de 5, 10, 20 e 30\%, e mais um grupo controle contendo apenas esta dieta.

Grupos de 100 ovos, coletados no mesmo dia, foram depositados em cada dieta e, sete dias após, as larvas de terceiro estágio foram retiradas, quantificadas, pesadas em balança analítica digital e transferidas para placas de Petri com papel filtro, com três gramas da dieta correspondente e três mililitros de água destilada, envolvidas com Parafilm com furos pequenos para aeração, mas que não permitissem sua saída. Após a formação das pupas, estas eram retiradas, quantificadas, pesadas em balança analítica digital e transferidas para placas de Petri sem substrato até a emergência dos adultos. Foram realizadas três repetições para cada grupo.

Os resultados foram submetidos à análise de variância e as médias foram comparadas entre si pelo teste de Tukey (SOKAL \& ROHLF, 1995) e regressões (PIMENTEL-GOMES, 2000; BANZATTO, 2008, utilizando o programa SAEG (UFV, 1999).

\section{RESULTADOS E DISCUSSÃO}

Os resultados descritos para viabilidade dos ovos são os que menos sofreram influência da temperatura, fato também observado nos trabalhos de KUNZ et al. (1977), que obtiveram $90 \%\left(23,9^{\circ} \mathrm{C}\right), 79 \%$ $\left(29,4^{\circ} \mathrm{C}\right)$ e $84,2 \%\left(35^{\circ} \mathrm{C}\right)$; MELLO; GARCIA (1988), $81,55 \%\left(27^{\circ} \mathrm{C}\right)$; VALGODE (1990), $91 \%\left(20^{\circ} \mathrm{C}\right), 95 \%$ $\left(25^{\circ} \mathrm{C}\right), 90 \%\left(30^{\circ} \mathrm{C}\right)$ e $86,5 \%\left(35^{\circ} \mathrm{C}\right)$; eLYSYK $(1998), 91 \%$ $\left(20^{\circ} \mathrm{C}\right), 93 \%\left(27^{\circ} \mathrm{C}\right)$ e $98 \%\left(35^{\circ} \mathrm{C}\right)$ de eclosão larval. Com base nesses valores apresentados, pode-se observar que, no presente estudo, em condições semelhantes às usadas por esses autores, a viabilidade foi menor do que a obtida pelos autores supracitados (Tabela 1).

O resultado obtido para viabilidade de ovos nas dietas com a substituição de $20 \%$ de água por vinhoto foi o mais próximo dos valores obtidos pelos autores citados. No entanto, pode-se observar que a substituição de $30 \%$ do volume de água por vinhoto na dieta influenciou negativamente na viabilidade dos ovos, e isso pode ser devido à alta demanda química de oxigênio e elevado conteúdo de sais minerais presentes no vinhoto (RABELO, 2010), que geram efeito tóxico no ambiente quando em excesso e podem dificultar o estabelecimento das larvas, que são o estádio mais crítico no desenvolvimento do inseto (MELLO, 1989).

Esses mesmos autores também avaliaram a viabilidade de larvas e apresentaram resultados similares entre si, a exemplo de KUNZ et al. (1977), que obtiveram $80,6 \%\left(23,9^{\circ} \mathrm{C}\right), 86,6 \%\left(29,4^{\circ} \mathrm{C}\right)$ e $66 \%\left(35^{\circ} \mathrm{C}\right)$; VALGODE $(1990), 53 \%\left(20^{\circ} \mathrm{C}\right), 70 \%\left(25^{\circ} \mathrm{C}\right)$ e $63 \%\left(30^{\circ} \mathrm{C}\right)$; e LYSYK $(1998), 83 \%\left(20^{\circ} \mathrm{C}\right), 65 \%\left(35^{\circ} \mathrm{C}\right)$ e $79 \%\left(27^{\circ} \mathrm{C}\right)$. Nesse parâmetro, mesmo os maiores valores obtidos pelos autores citados são inferiores aos obtidos em todos os tratamentos deste estudo. Apesar dos percentuais de viabilidade larval serem semelhantes, $o$ maior valor verificado foi novamente a partir da substituição de $20 \%$ de água por vinhoto. Os percentuais elevados podem estar relacionados à maior fonte de nutrientes disponibilizada na dieta pelo 
Tabela 1 - Média do número de larvas recuperadas de Stomoxys calcitrans, peso total e unitário em doses crescentes de vinhoto, a $27^{\circ} \mathrm{C}$ e $80 \%$ UR.

\begin{tabular}{lccc}
\hline Dose & Porcentagem de larvas recuperadas $(\%)$ & Peso total das larvas $(\mathrm{g})$ & Peso larva $^{-1}(\mathrm{~g})$ \\
\hline Controle & $66,0^{\mathrm{a}}$ & $1,0596^{\mathrm{a}}$ & $0,0164^{\mathrm{a}}$ \\
$5 \%$ & $64,0^{\mathrm{a}}$ & $1,0895^{\mathrm{a}}$ & $0,0169^{\mathrm{a}}$ \\
$10 \%$ & $69,3^{\mathrm{a}}$ & $1,3655^{\mathrm{a}}$ & $0,0197^{\mathrm{a}}$ \\
$20 \%$ & $80,0^{\mathrm{a}}$ & $1,2739^{\mathrm{a}}$ & $0,0158^{\mathrm{a}}$ \\
$30 \%$ & $20,3^{\mathrm{b}}$ & $0,3676^{\mathrm{b}}$ & $0,0180^{\mathrm{a}}$ \\
\hline
\end{tabular}

Nas colunas, médias seguidas de pelo menos uma mesma letra não diferem entre si pelo teste de Tukey (P>0,05).

vinhoto (SILVA et al., 2007). Desse modo, as larvas que eclodiram tiveram melhores condições de sobrevivência e, portanto, maior viabilidade, podendo, inclusive, explicar o aumento de moscas nas regiões produtoras de cana de açúcar (BARROS et al., 2010).

O estádio de pupa é o que sofre maior variação da sua viabilidade, segundo a temperatura, conforme pode ser observado nos estudos de KUNZ et al. (1977), os quais obtiveram uma média de $76,6 \%\left(23,9^{\circ} \mathrm{C}\right), 60,4 \%$ $\left(29,4^{\circ} \mathrm{C}\right)$ e $9,7 \%\left(35^{\circ} \mathrm{C}\right)$; nos de LYSYK $(1998)$, que obteve uma média de $93 \%\left(20^{\circ} \mathrm{C}\right), 78 \%\left(27^{\circ} \mathrm{C}\right)$ e $42 \%\left(35^{\circ} \mathrm{C}\right)$; e de VALGODE (1990), com 88,4\% $\left(20^{\circ} \mathrm{C}\right), 97 \%\left(25^{\circ} \mathrm{C}\right)$ e $96,6 \%$ $\left(30^{\circ} \mathrm{C}\right)$. No presente estudo, os valores obtidos são similares aos apresentados pelos autores citados. Entretanto, observou-se que, conforme se elevou a concentração de vinhoto na dieta, a viabilidade diminuiu, apesar da diferença dos resultados não ter sido significativa, visto que esse estádio é o que possui maior proteção frente às condições adversas do meio (MELLO, 1989) (Tabela 2).

Verificou-se que houve um aumento na produção de larvas e pupas, à medida que se elevou a dose de vinhoto, sugerindo que existe uma relação funcional da variável independente com efeito quadrático com ambas as variáveis dependentes. $\mathrm{Na}$ tabela 3, o número de moscas foi maior com o aumento da dose de vinhoto. $\mathrm{Na}$ avaliação do peso total das larvas, houve efeito significativo a $\mathrm{P}<0,05$, sendo menor para $30 \%$ de vinhoto e, para o peso unitário das larvas, não ocorreu diferença estatística (Tabela 1).Quanto ao peso unitário das larvas, não se obteve diferença estatística, no entanto, pode ser observado que o maior peso obtido foi na concentração de $10 \%$ de vinhoto, tanto para peso total quanto para peso unitário.

$\mathrm{Na}$ avaliação do período larval e do peso das pupas, houve efeito significativo a $\mathrm{P}<0.05$, em que o tratamento de $5 \%$ apresentou maior período em relação à concentração de 10 e $30 \%$ de vinhoto, demonstrando que o vinhoto a $5 \%$ dispersou o período de formação de pupas. O peso unitário pupal foi significativamente maior na dieta contendo $30 \%$ de vinhoto, em relação ao controle, 5 e $20 \%$ de vinhoto, mas não foi observada diferença estatística com a concentração de $10 \%$ de vinhoto (Tabela 2).

O período larval neste estudo foi semelhante ao verificado por autores em condições similares, tais como BAILEY et al. (1975), de 44 dias $\left(15^{\circ} \mathrm{C}\right), 9$ dias $\left(35^{\circ} \mathrm{C}\right)$ e 10 dias $\left(25^{\circ} \mathrm{C}\right)$ e MELLO (1989) de 15,9 dias $\left(18^{\circ} \mathrm{C}\right), 13,48$ dias $\left(27^{\circ} \mathrm{C}\right)$ e 14,6 dias (ambiente). Nas dietas acrescidas de $30 \%$ de vinhoto, pode ser observada concentração do período larval e maior peso pupal, possivelmente, por causa da maior disponibilidade de nutrientes na dieta e do menor número de indivíduos, pois era esperado que os

Tabela 2 - Média do período larval, viabilidade de larvas por dia, peso total e unitário das pupas de Stomoxys calcitrans em doses crescentes de vinhoto na dieta a $27^{\circ} \mathrm{C}$ e $80 \%$ UR.

\begin{tabular}{lcccc}
\hline Dose & Período larval (dias) & Número de pupas dia $^{-1}$ & Peso total das pupas $(\mathrm{g})^{\text {Peso pupa }^{-1}(\mathrm{~g})}$ \\
\hline Controle & $10,38^{\mathrm{ab}}$ & $37,5^{\mathrm{a}}$ & $0,3131^{\mathrm{ab}}$ & $0,0126^{\mathrm{b}}$ \\
$5 \%$ & $11,81^{\mathrm{a}}$ & $18,7^{\mathrm{b}}$ & $0,1780^{\mathrm{b}}$ & $0,0139^{\mathrm{b}}$ \\
$10 \%$ & $10,00^{\mathrm{b}}$ & $37,5^{\mathrm{a}}$ & $0,4224^{\mathrm{a}}$ & $0,0174^{\mathrm{ab}}$ \\
$20 \%$ & $10,86^{\mathrm{ab}}$ & $21,4^{\mathrm{ab}}$ & $0,2736^{\mathrm{ab}}$ & $0,0145^{\mathrm{b}}$ \\
$30 \%$ & $9,50^{\mathrm{b}}$ & $37,5^{\mathrm{a}}$ & $0,1451^{\mathrm{ab}}$ & $0,0219^{\mathrm{a}}$ \\
\hline
\end{tabular}

Nas colunas, médias seguidas de pelo menos uma mesma letra não diferem entre si pelo teste de Tukey (P>0,05). 
Tabela 3 - Média do período pupal e número de adultos de Stomoxys calcitrans emergidos em doses crescentes de vinhoto a $27^{\circ} \mathrm{C}$ e $80 \%$ UR.

\begin{tabular}{lcc}
\hline Dose & Período pupal (dias) & Número de moscas dia ${ }^{-1}$ \\
\hline Controle & $6,57^{\mathrm{ab}}$ & $14,3^{\mathrm{ab}}$ \\
$5 \%$ & $6,19^{\mathrm{b}}$ & $8,3^{\mathrm{b}}$ \\
$10 \%$ & $7,05^{\mathrm{a}}$ & $15,0^{\mathrm{ab}}$ \\
$20 \%$ & $7,00^{\mathrm{a}}$ & $10,7^{\mathrm{b}}$ \\
$30 \%$ & $6,00^{\mathrm{b}}$ & $18,8^{\mathrm{a}}$ \\
\hline
\end{tabular}

Nas colunas, médias seguidas de pelo menos uma mesma letra não diferem entre si pelo teste de Tukey $(\mathrm{P}>0,05)$.

indivíduos que sobrevivessem ao efeito tóxico do vinhoto fossem os mais resistentes e, portanto, apresentassem menor período larval, formando pupas mais rapidamente com mais reservas e mais pesadas.

$\mathrm{Na}$ avaliação do período pupal, houve efeito significativo a $\mathrm{P}<0.05 \mathrm{com}$ o maior período para os tratamentos 10 e $20 \%$ e menor para 5 e $30 \%$ de vinhoto (Tabela 3).

Os resultados de período pupal obtidos por Bailey et al. (1975) são de $20 \operatorname{dias}\left(15^{\circ} \mathrm{C}\right), 7 \operatorname{dias}\left(25^{\circ} \mathrm{C}\right)$ e 6 dias $\left(35^{\circ} \mathrm{C}\right)$. MELLO (1989) obteve de 35 a 36 dias $\left(18^{\circ} \mathrm{C}\right), 3,44$ dias $\left(27^{\circ} \mathrm{C}\right)$ e 3,1 dias (ambiente). O período pupal foi semelhante ao verificado por BAILEY et al. (1975) à temperatura de $25^{\circ} \mathrm{C}$, contudo foi menor nas concentrações de 5 e $30 \%$ de vinhoto. Pode ser observado que, na concentração de $30 \%$, houve uma emergência significativa de adultos, que pode estar relacionada ao maior peso das pupas formadas, as quais tinham mais reservas para sua sobrevivência.

\section{CONCLUSÃO}

Pode-se concluir que as maiores concentrações de vinhoto na dieta afetaram negativamente a viabilidade de ovos, enquanto que níveis mais baixos, favoreceram. A viabilidade de larvas foi aumentada nas concentrações intermediárias. O vinhoto não influenciou no peso das larvas. O período larval diminuiu à medida que se aumentou a sua concentração na dieta. O período pupal, o número de pupas formadas por dia, bem como o peso e o número total de pupas formadas não foram afetadas pela adição de vinhoto na dieta, contudo, a maior concentração de vinhoto aumentou a emergência de adultos.

\section{REFERÊNCIAS}

BAILEY, D.L. et al. Flight and dispersal of the stable fly. Journal of Economic Entomology, n.66, p.410-411, 1973.
BAILEY, D.L. et al. Laboratory biology and techniques for mass producing the Stable fly, Stomoxys calcitrans (L.) (Diptera: Muscidae). Journal of Medical Entomology, v.12, n.2, p.189-193, 1975.

BANZATTO, D.A.; KRONKA, S. do N. Experimentação agrícola. 4.ed. Jaboticabal: Afiliada, 2008. 237p.

BARROS, A.T.M. et al. Surtos por Stomoxys calcitrans em gado de corte no Mato Grosso do Sul. Pesquisa Veterinária Brasileira, v.30, n.11, p.945-952, 2010.

BENIGNO, R.N.M. Classificação etária fisiológica e comportamento alimentar de acordo com o sexo e desenvolvimento ovariano em Stomoxys calcitrans (L.) (Diptera: Muscidae). 1987. 96f. Dissertação (Mestrado em Parasitologia Veterinária) - Universidade Federal Rural do Rio de Janeiro, Seropédica, RJ.

BISHOPP, F.C. The stable fly (Stomoxys calcitrans: L.) an important livestock pest. Jornal of Economic Entomology, v.6, n.1, p.112-116, 1913

BITTENCOURT, A.J. Aspectos clinico - epidemiológicos de Stomoxys calcitrans (Linnaeus, 1758) em bovinos e eqüinos em Espírito Santo do Pinhal - SP. 1998. 120f. Tese (Doutorado em Parasitologia Veterinária) - Universidade Federal Rural do Rio de Janeiro, Seropédica, RJ.

BURALLI, G.M. et al. Soil disposal of residues and the proliferation of flies in the state of São Paulo. Water Science and Technology, v.19, n.8, p.121-125, 1987.

FURMAN, D.P.; CATTS, E.P. Manual of medical entomology. 4.ed. Cambridge: University, 1982. 207p.

GUIMARÃES, J.H. Moscas- Biologia, ecologia e controle. Agroquímica Ciba-Geigy, n.21, p.20-26,1983.

HOGSETTE, J.A.; RUFF, J.P. Stable fly (Diptera: Muscidae) migration in northwest Florida. Environmental Entomology, n.14, p.170-175, 1985.

KING, W.V.; LENERT, L.G. Outbreaks of Stomoxys calcitrans L. ('dog flies') along Florida's northwest coast. Florida Entomologist, v.19, n.3, p.33-39, 1936.

KUNZ, S.E. et al. The development of the immature forms of Stomoxys calcitrans. Annals of the Entomological Society of America, v.70, n.2, p.169-172, 1977.

LYSYK, T.J. Relationships between temperature and life-history parameters of Stomoxys calcitrans (Diptera: Muscidae). Journal of Medical Entomology, v.35, n.2, p.107-119, 1998.

MELLO, R.P.; GARCIA, M.L.M. Comportamento reprodutivo de fêmeas de Stomoxys calcitrans (L.) (Diptera: Muscidae) criadas isoladamente em laboratório. Memórias do Instituto Oswaldo Cruz, v.83, n.3, p.385-390, 1988.

MELLO, R.P. Estudos de alguns aspectos de desenvolvimento biológico e do comportamento, em laboratório, de Stomoxys calcitrans, (Linnaeus, 1758) (Diptera: Muscidae). 1989. 141f. Tese (Doutorado em Medicina Veterinária - Parasitologia Veterinária) - Universidade Federal Rural do Rio de Janeiro, Seropédica, RS. 
MORAES, J.L.C. Toxicidade comparativa de alguns inseticidas organofosforados e piretróides sobre larvas e adultos de Stomoxys calcitrans Linnaeus, 1758. 1990.

61f. Dissertação (Mestrado em Ciências - Parasitologia Veterinária) - Universidade Federal Rural do Rio de Janeiro, Seropédica, RJ.

PIMENTEL-GOMES, F. Curso de estatística experimental. 14.ed. Piracicaba: Degaspari, 2000. 477p.

RABELO, S.C. Aproveitamento de resíduos industriais. In: SANTOS, F. et al. Cana-de-Açúcar: bioenergia, açúcar e álcool - tecnologia e perspectivas. Viçosa: Celso Caldas, 2010. Cap.17, p.465-486.

SILVA, M.A.S. et al. Uso de vinhaça e impactos nas propriedades do solo e lençol freático. Revista Brasileira de Engenharia Agrícola e Ambiental, v.11, n.1, p.108-114, 2007.
SOKAL, R.R.; ROHLF, J. Biometry: the principles and practice of statistics in biological research. 3.ed. ew York: W.H. Freeman, 1995

SOULSBY, E.J.L. Parasitología y Enfermidades parasitarias en los animales domésticos. 7.ed. México: Nova Editorial Interamericana, 1987. 823p.

UNIVERSIDADE FEDERAL DE VIÇOSA. Sistema de análises estatísticas e genéticas: SAEG. Viçosa: 1999. 138p. (Manual do usuário, versão 8.0).

VALGODE, M.A. Exigências térmicas e temperatura base das formas imaturas de Stomoxys calcitrans (Linnaeus, 1758) (Diptera: Muscidae). 1990. 67f. Dissertação (Mestrado em Parasitologia Veterinária) - Universidade Federal Rural do Rio de Janeiro, Seropédica, RJ. 